Louisiana State University

LSU Digital Commons

$1-1-2014$

\title{
MRI investigation of the linkage between respiratory motion of the heart and markers on patient's abdomen and chest: Implications for respiratory amplitude binning list-mode PET and SPECT studies
}

\author{
Paul Dasari \\ University of Massachusetts Medical School \\ Karen Johnson \\ University of Massachusetts Medical School \\ Joyoni Dey \\ University of Massachusetts Medical School \\ Clifford Lindsay \\ University of Massachusetts Medical School \\ Mohammed S. Shazeeb \\ University of Massachusetts Medical School
}

See next page for additional authors

Follow this and additional works at: https://digitalcommons.Isu.edu/physics_astronomy_pubs

\section{Recommended Citation}

Dasari, P., Johnson, K., Dey, J., Lindsay, C., Shazeeb, M., Mukherjee, J., Zheng, S., \& King, M. (2014). MRI investigation of the linkage between respiratory motion of the heart and markers on patient's abdomen and chest: Implications for respiratory amplitude binning list-mode PET and SPECT studies. IEEE Transactions on Nuclear Science, 61 (1), 192-201. https://doi.org/10.1109/TNS.2013.2294829

This Article is brought to you for free and open access by the Department of Physics \& Astronomy at LSU Digital Commons. It has been accepted for inclusion in Faculty Publications by an authorized administrator of LSU Digital Commons. For more information, please contact ir@lsu.edu. 


\section{Authors}

Paul Dasari, Karen Johnson, Joyoni Dey, Clifford Lindsay, Mohammed S. Shazeeb, Joyeeta Mitra Mukherjee, Shaokuan Zheng, and Michael A. King 


\title{
MRI Investigation of the Linkage Between Respiratory Motion of the Heart and Markers on Patient's Abdomen and Chest: Implications for Respiratory Amplitude Binning List-Mode PET and SPECT Studies
}

\author{
Paul Dasari, \\ Department of Radiology, University of Massachusetts Medical School, Worcester, MA 01655 \\ USA and also with the Department of Biomedical Engineering, Worcester Polytechnic Institute, \\ Worcester, MA 01609 USA (paulkreddy@wpi.edu).

\section{Karen Johnson,} \\ Department of Radiology, University of Massachusetts Medical School, Worcester, MA 01655 \\ USA. \\ Joyoni Dey [Member, IEEE], \\ Department of Radiology, University of Massachusetts Medical School, Worcester, MA 01655 \\ USA. \\ Clifford Lindsay, \\ Department of Radiology, University of Massachusetts Medical School, Worcester, MA 01655 \\ USA.
}

\section{Mohammed S. Shazeeb,}

Department of Radiology, University of Massachusetts Medical School, Worcester, MA 01655 USA.

Joyeeta Mitra Mukherjee [Member, IEEE],

Department of Radiology, University of Massachusetts Medical School, Worcester, MA 01655 USA.

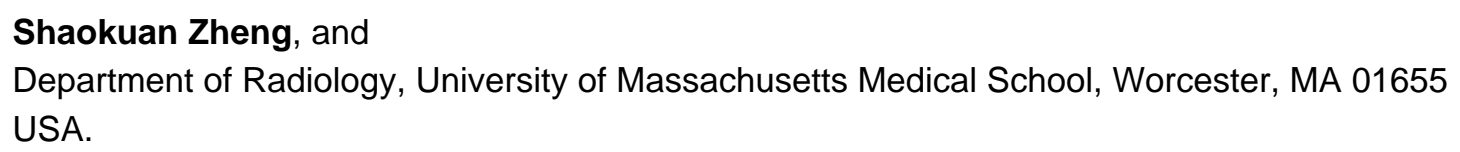

\begin{abstract}
Respiratory motion of the heart impacts the diagnostic accuracy of myocardial-perfusion emission-imaging studies. Amplitude binning has come to be the method of choice for binning
\end{abstract}


list-mode based acquisitions for correction of respiratory motion in PET and SPECT. In some subjects respiratory motion exhibits hysteretic behavior similar to damped non-linear cyclic systems. The detection and correction of hysteresis between the signals from surface movement of the patient's body used in binning and the motion of the heart within the chest remains an open area for investigation. This study reports our investigation in nine volunteers of the combined MRI tracking of the internal respiratory motion of the heart using Navigators with stereo-tracking of markers on the volunteer's chest and abdomen by a visual-tracking system (VTS). The respiratory motion signals from the internal organs and the external markers were evaluated for hysteretic behavior analyzing the temporal correspondence of the signals. In general, a strong, positive correlation between the external marker motion (AP direction) and the internal heart motion (SI direction) during respiration was observed. The average \pm standard deviation in the Spearman's ranked correlation coefficient $(\rho)$ over the nine volunteer studied was $0.92 \pm 0.1$ between the external abdomen marker and the internal heart, and $0.87 \pm 0.2$ between the external chest marker and the internal heart. However despite the good correlation on average for the nine volunteers, in three studies a poor correlation was observed due to hysteretic behavior between inspiration and expiration for either the chest marker and the internal motion of the heart, or the abdominal marker and the motion of the heart. In all cases we observed a good correlation of at least either the abdomen or the chest with the heart. Based on this result, we propose the use of marker motion from both the chest and abdomen regions when estimating the internal heart motion to detect and address hysteresis when binning list-mode emission data.

\section{Keywords}

Cardiac respiratory motion; emission tomography; hysteresis; MRI; signal processing

\section{Introduction}

RESPIRATORY motion and other body motions in cardiac-perfusion PET and SPECT imaging are inevitable due to the long scan duration (typically 3-15 mins) [1], [2]. The effects of motion during imaging result in blurring and mismatch between emission and transmission / CT imaging for estimation of attenuation maps. These in turn degrade the accuracy of the diagnostic imaging.

Motion can be reduced by using some respirator-surrogate signal to select for acquisition just the data acquired near end-expiration, when one typically pauses momentarily [3]. However, this can trade an increase in noise due to a reduction in counts acquired or require a prolongation of acquisition for improved resolution due to less motion being present. With use of list-mode acquisition, all the counts can be acquired and combined into motion states (bins) post-acquisition based on a simultaneously acquired signal related to the extent of respiration. Use of the amplitude of this signal has been shown to result in more accurate and consistent binning than use of the phase for correction of respiratory motion [4]-[6]. It has been demonstrated that the respiratory motion of the diaphragm is correlated with the anterior / posterior motion of the abdomen [7]. Thus, the internal motion of the diaphragm can be predicted from an external respiratory signal. The respiratory motion of the heart results primarily from the motion of the diaphragm in the superior-inferior (SI) direction and 
rocking of the ribs causing anterior-posterior (AP) motion [8], [9]. However, the relationship between the amplitude of the external signal and the actual position of internal structures can be complex.

A number of studies have been performed using MRI and CT to investigate and model the respiratory motion of various anatomical regions. The most common regions investigated have been the lung [10]-[14], diaphragm [7], [15], and heart [9], [16], [17]. For example, Nehrke et al. [9] reported on the presence of hysteresis between the displacements of the heart and the diaphragm during free breathing using a multi-navigator echo technique in MRI.

The aim of this study was to follow-up on the investigations of Nehrke et al. [9] with the goal of better understanding the relationship between internal motion of the heart and external motion of surrogates during respiratory motion using MRI and a visual-tracking system (VTS) [18], respectively. This was done by motion-tracking external markers on the abdomen and chest simultaneously with MRI tracking of respiratory motion the heart, chest, and dome of the liver. An analysis of the correlation between the external and internal motion of these locations was then employed to further explore the relationship between external signals and motion of the heart.

\section{Materials and Methods}

The VTS employed in these investigations allows near-infrared tracking of multiple markers within the 3-dimensional imaging volume of the MRI [18]. The VTS is comprised of the following components: the infrared cameras, the controlling hardware module, and the software to analyze and present the data. Three cameras of the VTS system were mounted on the wall of the MRI room adjusted such that the center of the volume to be tracked is at the iso-center of the MRI. Before each experimental session the VTS system is calibrated for stereo motion-tracking and tested for minimal RF interference with the MRI as discussed in [19]. Stereo imaging of the external retro-reflective markers on the thoracic-abdominal regions of volunteers by VTS was performed at 30 frames per second throughout MRI imaging. Synchronization between the MR scanner and VTS system was established by using a trigger signal from the MR scanner at the beginning of acquisition to start motion capture by the VTS system.

Nine healthy volunteers without counter-indications to MRI were studied in this investigation. One of the volunteers was investigated on two different occasions. The participation of volunteers was with IRB approval and informed consent. The volunteers were prepared for VTS imaging by wrapping bands with 7 markers about their chest and abdomen as illustrated in Fig. 1(a). The markers were $2 \mathrm{~cm}$ diameter retro-reflectively coated hollow spheres filled with copper sulfate solution. The marker positions were those employed clinically for robust motion tracking and correction in our clinic [18]. They were derived over several years of clinical usage. During imaging the volunteers were positioned supine on the table of the MRI (Fig. 1(b)). Their hands were over their heads on the same support as employed during cardiac SPECT imaging in our clinic, and their feet faced the 
VTS. They were instructed to breathe slowly and regularly over the approximately 5-minute period during which MRI and motion-tracking were performed.

MRI was performed using a Philips Healthcare ACHIEVA 3.0-T whole body MR scanner. The MR software provided for acquisition by three independent pencil-beam navigator radiofrequency pulses (navigators)[20]. These could be freely placed in space at any desired location and angle as guided by patient anatomy portrayed in Survey slices acquired at the start of each imaging session. Read-out gradients in the direction of the pencil-beams acquire the navigator echo signals. The Fourier transform of the signal produces the projection of the magnetization in the motion direction (read-out direction) on to the navigator profile. This is displayed as an M mode image (Fig. 2). Real-time displacement of the anatomic region along the direction of the pencil-beam was determined by performing cross-correlational analysis between the current navigator profile and the previous reference profile [21].

Using Survey slices of the volunteer for guidance the three navigator windows were positioned at the selected locations of the volunteers as illustrated in Fig. 2. Shown there are transverse, coronal and sagittal slices with the location of one or two of the three windows superimposed on each slice. The first of the navigator windows was positioned on the superior left-ventricular wall/lung boundary. As illustrated in the Fig. 2, this provided tracking of the ventricular wall in the SI direction, which is the direction of the largest component of cardiac respiratory motion [8], [9]. The second navigator window was on the dome of the right diaphragm. It too was positioned to track the SI motion of this structure as a relationship between diaphragm motion and the SI motion of the heart has been well established [8], [9]. Also it has been shown that there is an excellent correlation between the motion of the diaphragm and that of an external surrogate [7]. Thus the motion obtained from this navigator was employed to validate the AP motion of the abdominal markers as determined by the VTS. The third navigator window was positioned on the anterior wall of the chest to track its AP motion. The purpose of this navigator was to validate the AP motion determined from the chest markers of the VTS. The location of the tissue interfaces in these navigator beams were stored in real-time in list format in the scanner software for further analysis.

In addition to this multi-navigator sequence, dynamic acquisition of 300 single sagittal slices through the left ventricle (LV) of the heart was acquired to provide an independent measure from the navigator methodology of the respiratory motion of the heart.

MRI was performed during continuous breathing using the ECG-triggered single shot, 2D Fast-Field-Echo (Gradient Echo) sequence, with TR/TE $=5.5 / 2.1 \mathrm{~ms}, 128 \times 128$ image matrix, and a $250 \mathrm{~ms}$ acquisition window at mid-diastole. The MRI acquisitions also included recording the signal from a bellows (pressure sensor) on the volunteer's abdomen and the EKG.

The signal for the location of the tissue interface recorded from each of the internal navigators and the vertical motion of the external markers were separated into inspiration and expiration phases based on peak detection. Selected pair-wise comparisons of 
respiratory signals from thoracic and abdomen regions were plotted against each other as a function of time to visually look for spatial and temporal correlations respectively between the signals. Statistically the signals were checked for correlations through calculation of parametric (linear) and non-parametric (ranked) measures of correlation. A Pearson productmoment correlation coefficient $(r)$ [22] was computed to assess the strength of a linear association in the relationship between amount of displacement of the chest and abdominal markers, and of the heart and the diaphragm navigators. The Spearman rank-order correlation coefficient $(\rho)$ [23] was computed as a non-parametric measure of the strength and direction of association between the external AP motion of the markers and the internal SI motion of the heart. The chest wall navigator was used along with the external VTS marker to study the correlation strength between the two modalities by computing Spearman rank-order correlation coefficient $(\rho)$.

The MRI sagittal slices from the dynamic acquisitions were used in determining the extent of heart motion in terms of SI translation, AP translation, and rotation about the lateral axis using semi-automatic segmentation and registration [24]. This was done by using a 2-D semi-automated segmentation algorithm that segments the heart in the dynamic MRI datasets for estimating the respiratory motion of the heart. The segmented first dynamic slice was used in 3 degree-of-freedom (DOF) affine registration to estimate the respiratory motion relative to it for each subsequent slice. The translation motion of the heart in the SI direction obtained from the registration algorithm was compared against that of the heart navigator to validate these measurements. The VTS marker data was re-sampled to match the sampling rate of the heart navigator in order to perform correlation analysis between the external markers and the internal motion of the heart.

The respiratory signal from the bellows on the volunteer's abdomen was recorded but was not used in this study. All data analysis was performed in MATLAB (R2011b, Mathworks, Natick, MA).

\section{Results}

Showing all the results from our nine volunteers is prohibited by the amount of space that would be required. Thus we will show the results of selected example volunteer studies herein. Each volunteer was assigned a number for the purpose of this paper and this volunteer number will be provided when results are given.

VTS chest markers and the chest wall navigator showed a strong, positive correlation with an average \pm standard deviation of $0.95 \pm 0.02$ for nine volunteers. The heart motion obtained from the navigators and the slices through registration of the segmented heart also showed a strong, positive correlation with an average \pm standard deviation of $0.96 \pm 0.02$ for nine volunteers. Thus we were able to validate through the comparison of these two independently derived measures the usage of the Navigator signal for tracking the external motion of the chest wall and for usage in tracking the SI motion of the superior wall of the LV internally. Herein we will use the Navigator measured internal motion of the heart and the VTS externally measured motion of chest as our comparisons of tracked motions. 
Example results of the data from external markers and internal navigators for Volunteer 3 are provided in Fig. 3. Notice the strong similarity of motion between the five chest markers and between the two abdomen markers for this volunteer. This trend was seen for all nine volunteers as exemplified by the strong pair-wise correlation over all-pairs of comparison between the motions of the five chest markers $(0.97 \pm 0.02)$ and the two abdomen markers $(0.98 \pm 0.01)$. This implies that any of the chest markers or either of the abdomen markers could be used for comparison to the navigator measured internal motions. This result is further illustrated in Fig 4, which shows plots of the individual chest and abdomen Spearman ranked correlations of the motion of individual external markers with the navigator internal heart motion for nine volunteers. Notice that the extent of external to internal agreement does vary with volunteers, but is quite similar between markers for a given volunteer. Thus we will show the results for marker 3 on the chest and marker 1 on the abdomen (seen Fig 1(a) for marker location) in the rest of our results presented herein.

Examples of the agreement found between VTS determined AP displacements of the external chest and abdomen markers with the internal SI respiratory motion of the heart superior LV wall as determined by the navigator for 3 volunteers are shown in Fig 5. The Spearman ranked correlation coefficients between the internal heart motion and the VTS chest and abdomen markers are also given to provide a quantitative measure of the correlation in the shapes (but not actual magnitudes) of the motions. The plots of Fig. 5(a) visually demonstrate a well-defined temporal correlation between the internal and the external motions despite the irregularity in this volunteer's respiration. The visual impression of good agreement was supported by the correlation coefficients obtained for this study.

In general over the nine volunteers, a strong, positive correlation between the external marker motion (AP direction) and the internal heart motion (SI direction) during expiration and inspiration phases was observed. The average \pm standard deviation in the Spearman's ranked correlation coefficient $(\rho)$ over the nine volunteer studies was $0.95 \pm 0.1$ between the external abdomen marker and the internal heart, and $0.87 \pm 0.2$ between the external chest marker and the internal heart. Though generally good, not all studies showed a strong correlation to both abdomen and chest markers. This is illustrated in Fig. 5(b), which shows the time series plot for a volunteer with moderate agreement $(\rho=0.78)$ between the abdomen marker and the heart, and a strong correlation between the chest marker and the heart $(\rho=0.96)$. In Fig. 5(c) is shown an example of a poor correlation $(\rho=0.38)$ between the chest marker and the heart, and a strong agreement between the abdomen and the heart $\rho$ $=0.88$ ). Thus we have observed either the chest or the abdomen marker signals to not correlate strongly with the motion of the heart; however, for the limited number of volunteers in this study, we have not encountered a case where both the chest markers and the abdomen markers were poorly correlated with the heart motion.

It is difficult to visualize the differences in the motion of the heart versus the external tracking of motion in Fig. 5(b) and (c). This is better seen in the 2D scatter plots shown in Fig. 6, which provides results for measurements in these two volunteers plus three more of our nine volunteers. As illustrated on the left in this figure there was in many but not all cases a strong, positive correlation between the internal motions of the heart and the 
diaphragm. The average \pm standard deviation in the Pearson correlation coefficient $(r)$ over all nine volunteer studies was $0.87 \pm 0.13$. On the right of this figure is shown the corresponding external motions of the chest and abdomen markers, which also generally show a strong, positive correlation. The average \pm standard deviation in the Pearson correlation coefficient was $0.82 \pm 0.21$.

As shown in the plots of Fig. 6 not all volunteers exhibited a monotonic relationship between the two tracked motions. Some of the plots show hysteresis, a difference in trajectories between inspiration and expiration as seen with volunteers 3 and 4 . Note that the extent of hysteresis is highly volunteer dependent. In this study of nine volunteers, six showed a linear or monotonic relationship visually for both the internal and external measurements. Further these six had common trajectories in the 2D plots for both inspiration and expiration phases as illustrated in Fig. 6(a) and (b). The scatter plots for two other volunteers shown in Fig. 6(c) and (d), exhibited moderate to strong hysteresis for both the internal and external measurements, with distinct trajectories for inspiration and expiration. For one case shown in Fig. 6(e) the 2D scatter plot for external marker motions of the chest and abdomen showed hysteretic loops drifting downward, while the corresponding scatter plot for internal motion exhibited a monotonic trend. The direction of the hysteretic loops is always counter-clockwise, but with varying degree of hysteresis for every respiratory cycle. The time series plots for this volunteer were shown in Fig. 5(c).

The 2D scatter plots of Fig. 7 provide a direct comparison between the external markers (chest and abdomen) and the internal respiratory motion of the heart for examples of linear and hysteretic motion. The linear case is shown in Fig. 7(a) and is from volunteers 2. Note that the motion of both the chest and abdomen markers correlates linearly with the internal motion of the heart. The hysteretic case shown in Fig 7(b) is from volunteer 4. Note the linear correspondence between the chest motion and internal heart motion; however, the correlation between the abdomen motion and the internal heart motion was poor, which is in good agreement with the results seen in Fig. 6(d).

Fig. 8 shows the original and repeat study at a later date for Volunteer 4 that showed the greatest extent of hysteresis. From the plots we see that the volunteer exhibited hysteretic pattern both internally and externally when deeper breathing was performed; on the other hand, the respiratory curves on the scatter plots showed a linear trend for shallow breathing. This indicated that the hysteretic effect is not the characteristic of this individual but rather could manifest randomly, i.e. it not only varies between volunteers but in time (or with extent of respiration) in a given volunteer.

\section{Discussion}

The irregular breathing patterns along with the hysteresis observed in our studies may have significant impact on the accuracy and reliability of the gating / binning methods used in respiratory-motion correction in emission tomography when imaging the heart and other structure in the chest and abdomen. It can also impact the formation of 4D-CT studies [4], [5], [25], [26] and patient treatment in radiation therapy [10], [14], [27]-[29]. As illustrated in Fig. 6(c) and (d), with external-signal amplitude based binning of list-mode studies, when 
hysteresis is present a fixed range of displacement of the abdomen marker can corresponds to a deviation in displacement of the heart for expiratory and inspiratory respiratory phases. Performing amplitude binning in such a study would result in considerable variation in the SI location of the heart in the list-mode events placed in that bin. This would result in an incomplete correction of respiratory motion.

In addition to the hysteresis, variation in respiratory amplitude and baseline as has shown in Figs. 5(c) and 6(e) presents another complication when performing amplitude binning. This can result in incorrect sorting of spatial positions of the heart for a given amplitude bin eventually affecting the diagnostic accuracy of the images. Such variability has been determined to be present in patients undergoing PET/CT [30] and SPECT [31] imaging.

The most direct approach to tackle the problem of hysteresis is to treat the inspiration and expiration phases separately [4]. However, the resulting images are subjected to poor signalto-noise ratio since each of the frames contains only part of the counts available throughout the acquisition of a respiration average emission dataset therefore yielding noisy images. Alternatively, since the pattern of hysteresis if present manifests itself both internally and externally one could combine the respiratory information acquired from the chest or the abdomen areas with a motion model that takes hysteresis into account when predicting the motion of the heart. Conceivably, from the examples presented in this study, using the respiratory information from both the chest and the abdomen regions, comparable to the work shown by Fayad et al. [32] and by Odille et al. [33], along with a better motion model to account for both hysteresis and irregular breathing patterns would result in more accurate respiratory motion correction. An excellent recent review of respiratory motion modeling provides more details on the current status of such modeling in medical imaging [34].

We are uncertain of the physiological basis for our observations that the presence of hysteresis of heart motion internally is manifested externally in a difference in the chest and abdomen marker motion. One possible explanation is the following. There are two principle mechanisms for changing respiratory volume of the lungs. The first is that of the SI motion of the diaphragm, which would result in AP motion of markers on the abdomen as demonstrated by Vedam et al. [7]. The second is a rocking of the ribs, which expands the circumference of the chest [35], thereby causing AP motion of chest markers. The interplay of these with the viscoelastic nature of the lungs [9] and possible internal imbalances in lung pressure [36] could play a part in retarding the internal motion of the heart. Such a damping could then result in delayed periodic motion as observed in a forced damped oscillation [37]. However study of a much larger population of volunteers including those with lung pathologies is needed to further clarify the physiological basis for our findings.

\section{Conclusion}

In this study, we investigated the correspondence of the respiratory motion of the heart as assessed internally using the Navigator methodology to the external motion of markers measured by a VTS. We determined hysteresis and irregular motion to be present in several of our volunteers. The hysteresis between the markers of the chest and the abdomen correlates with SI motion of the heart and the diaphragm. It is thus potentially a means to 
indicate the presence of hysteresis and could be useful in predicting the respiratory motion of the heart. The motion of the ensemble of the markers combined with a better motion model may be useful in providing an improved strategy for binning list-mode emission data into more accurate, consistent binning. Our future work will involve the development of a suitable motion model to account for hysteresis and irregular breathing, and then to study the performance of the developed model on phantom simulations and clinical studies.

\section{Acknowledgments}

This work was supported by the National Institute of Biomedical Imaging and Bioengineering (NIBIB) under grant R01 EB001457 and a grant from Philips Healthcare. The contents are solely the responsibility of the authors and do not necessarily represent the official views of the NIBIB or Philips Healthcare.

\section{References}

[1]. Dilsizian V, Bacharach S, Beanlands R, Bergmann S, Delbeke D, Gropler R, et al. PET myocardial perfusion and metabolism clinical imaging. J. Nucl. Cardiol. 2009; 16:651-51.

[2]. Holly TA, Abbott BG, Al-Mallah M, Calnon DA, Cohen MC, DiFilippo FP, et al. Single photonemission computed tomography. J. Nucl. Cardiol. 2010; 17:941-73. [PubMed: 20552312]

[3]. Cho K, Kumiata S, Okada S, Kumazaki T. Development of respiratory gated myocardial SPECT system. J. Nucl. Cardiol. 1999; 6:20-28. [PubMed: 10070837]

[4]. Olsen JR, Lu W, Hubenschmidt JP, Nystrom MM, Klahr P, Bradley JD, et al. Effect of novel amplitude/phase binning algorithm on commercial four-dimensional computed tomography quality. Int. J. Radiat. Oncol. Biol. Phys. 2008; 70:243-52. [PubMed: 18037590]

[5]. Abdelnour AF, Nehmeh SA, Pan T, Humm JL, Vernon P, Schoder H, et al. Phase and amplitude binning for 4D-CT imaging. Phys. Med. Biol. 2007; 52:3515-29. [PubMed: 17664557]

[6]. Dawood M, Buther F, Lang N, Schober O, Schafers KP. Respiratory gating in positron emission tomography: A quantitative comparison of different gating schemes. Med. Phys. 2007; 34:306776. [PubMed: 17822014]

[7]. Vedam SS, Kini VR, Keall PJ, Ramakrishnan V, Mostafavi H, Mohan R. Quantifying the predictability of diaphragm motion during respiration with a noninvasive external marker. Med. Phys. 2003; 30:505-13. [PubMed: 12722802]

[8]. McLeish K, Hill DL, Atkinson D, Blackall JM, Razavi R. A study of the motion and deformation of the heart due to respiration. IEEE Trans. Med. Imaging. 2002; 21:1142-50. [PubMed: 12564882]

[9]. Nehrke K, Bornert P, Manke D, Bock JC. Free-breathing cardiac MR imaging: Study of implications of respiratory motion-initial results. Radiology. 2001; 220:810-15. [PubMed: 11526286]

[10]. Seppenwoolde Y, Shirato H, Kitamura K, Shimizu S, van Herk M, Lebesque JV, et al. Precise and real-time measurement of 3D tumor motion in lung due to breathing and heartbeat, measured during radiotherapy. Int. J. Radiat. Oncol. Biol. Phys. 2002; 53:822-34. [PubMed: 12095547]

[11]. Blackall JM, Ahmad S, Miquel ME, McClelland JR, Landau DB, Hawkes DJ. MRI-based measurements of respiratory motion variability and assessment of imaging strategies for radiotherapy planning. Phys. Med. Biol. 2006; 51:4147-69. [PubMed: 16912374]

[12]. Chi PC, Balter P, Luo D, Mohan R, Pan T. Relation of external surface to internal tumor motion studied with cine CT. Med. Phys. 2006; 33:3116-23. [PubMed: 17022203]

[13]. Liu C, Alessio AM, Kinahan PE. Respiratory motion correction for quantitative PET/CT using all detected events with internal-external motion correlation. Med. Phys. 2011; 38:2715-23. [PubMed: 21776808]

[14]. Ruan D, Fessler JA, Balter JM, Berbeco RI, Nishioka S, Shirato H. Inference of hysteretic respiratory tumor motion from external surrogates: A state augmentation approach. Phys. Med. Biol. 2008; 53:2923-36. [PubMed: 18460744] 
[15]. McQuaid SJ, Lambrou T, Hutton BF. A novel method for incorporating respiratory-matched attenuation correction in the motion correction of cardiac PET-CT studies. Phys. Med. Biol. 2011; 56:2903-15. [PubMed: 21490383]

[16]. Burger I, Meintjes EM. Elliptical subject-specific model of respiratory motion for cardiac MRI. Magn. Reson. Med. 2012

[17]. Dasari, P.; Johnson, K.; Dey, J.; Mukherjee, JM.; Shaokuan, Z.; Connolly, C., et al. MRI investigation of the linkage between respiratory motion of the heart and markers on patient's abdomen and chest: Implications for respiratory amplitude binning list-mode PET and SPECT studies. Proc. IEEE Nucl. Sci. Symp. Conf. Rec. (NSS/MIC); Valencia, Spain. 2011. p. 3748-51.

[18]. McNamara JE, Pretorius PH, Johnson K, Mukherjee JM, Dey J, Gennert MA, et al. A flexible multicamera visual-tracking system for detecting and correcting motion-induced artifacts in cardiac SPECT slices. Med. Phys. 2009; 36:1913-23. [PubMed: 19544811]

[19]. King, MA.; Dey, J.; Johnson, K.; Dasari, P.; Mukherjee, J.; Mc-Namara, J., et al. Use of MRI to assess the prediction of heart motion by stereo-tracking of markers on the body surface. Proc. IEEE Nucl Sci Symp Conf Rec (NSS/MIC); Knoxville, TN, USA. 2010. p. 3320-25.

[20]. Ehman RL, Felmlee JP. Adaptive technique for high-definition MR imaging of moving structures. Radiology. 1989; 173:255-63. [PubMed: 2781017]

[21]. Wang Y, Grimm RC, Felmlee JP, Riederer SJ, Ehman RL. Algorithms for extracting motion information from navigator echoes. Magn. Reson. Med. 1996; 36:117-23. [PubMed: 8795030]

[22]. Gibbons, S. C. Jean Dickinson Nonparametric Statistical Inference. 5th ed. Chapman and Hall; London, U.K.: 2010.

[23]. Best DJ, Roberts DE. Algorithm AS 89: The upper tail probabilities of Spearman's rho. J. Roy. Stat. Soc. C-App. 1975; 24:377-79.

[24]. Dey J, Pan T, Choi DJ, Robotis D, Smyczynski MS, Pretorius PH, et al. Estimation of cardiac respiratory-motion by semi-automatic segmentation and registration of non-contrast-enhanced 4D-CT cardiac datasets. IEEE Trans. Nucl. Sci. 2009; 56:3662-71. [PubMed: 20419041]

[25]. Pan T, Lee TY, Rietzel E, Chen GT. 4D-CT imaging of a volume influenced by respiratory motion on multi-slice CT. Med. Phys. 2004; 31:333-40. [PubMed: 15000619]

[26]. Lu W, Parikh PJ, Hubenschmidt JP, Bradley JD, Low DA. A comparison between amplitude sorting and phase-angle sorting using external respiratory measurement for 4D CT. Med. Phys. 2006; 33:2964-74. [PubMed: 16964875]

[27]. Webb S. Motion effects in (intensity modulated) radiation therapy: A review. Phys. Med. Biol. 2006; 51:R403-25. [PubMed: 16790915]

[28]. Barnes PJ, Baldock C, Meikle SR, Fulton RR. Benchmarking of a motion sensing system for medical imaging and radiotherapy. Phys. Med. Biol. 2008; 53:5845-57. [PubMed: 18827318]

[29]. Seppenwoolde Y, Berbeco RI, Nishioka S, Shirato H, Heijmen B. Accuracy of tumor motion compensation algorithm from a robotic respiratory tracking system: A simulation study. Med. Phys. 2007; 34:2774-84. [PubMed: 17821984]

[30]. Kinahan P, Wollenweber S, Alessio A, Kohlmyer S, MacDonald L, Lewellen T, et al. Impact of respiration variability on respiratory gated whole-body PET/CT imaging. J. Nucl. Med. 2007; 48:196P. Meeting Abstracts.

[31]. Mukherjee JM, Johnson KL, McNamara JE, King MA. Quantitative study of rigid-body and respiratory motion of patients undergoing stress and rest cardiac SPECT imaging. IEEE Trans. Nucl. Sci. 2010; 57:1105-15. [PubMed: 20694041]

[32]. Fayad H, Pan T, Pradier O, Visvikis D. Patient specific respiratory motion modeling using a 3D patient's external surface. Med. Phys. 2012; 39:3386-95. [PubMed: 22755719]

[33]. Odille F, Cindea N, Mandry D, Pasquier C, Vuissoz PA, Felblinger J. Generalized MRI reconstruction including elastic physiological motion and coil sensitivity encoding. Magn. Reson. Med. Jun.2008 59:1401-11. [PubMed: 18421689]

[34]. McClelland JR, Hawkes DJ, Schaeffter T, King AP. Respiratory motion models: A review. Med. Image. Anal. 2013; 17:19-42. [PubMed: 23123330]

[35]. Martin, DE.; Youtsey, JW. Respiratory Anatomy and Physiology. C. V. Mosby; St. Louis, MO, USA: 1988. 
[36]. Low DA, Parikh PJ, Lu W, Dempsey JF, Wahab SH, Hubenschmidt JP, et al. Novel breathing motion model for radio-therapy. Int. J. Radiat. Oncol. Biol. Phys. 2005; 63:921-9. [PubMed: 16140468]

[37]. Gutiérrez E, Paulotto C. Delay correlation of mechanical hysteresis and damping. J. Struct. Eng. 2009; 136:76-85. 


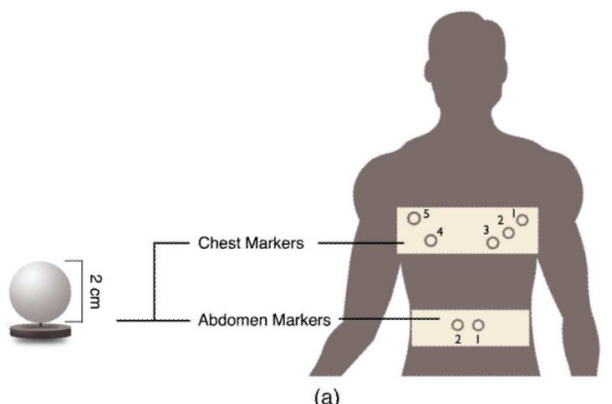

(a)

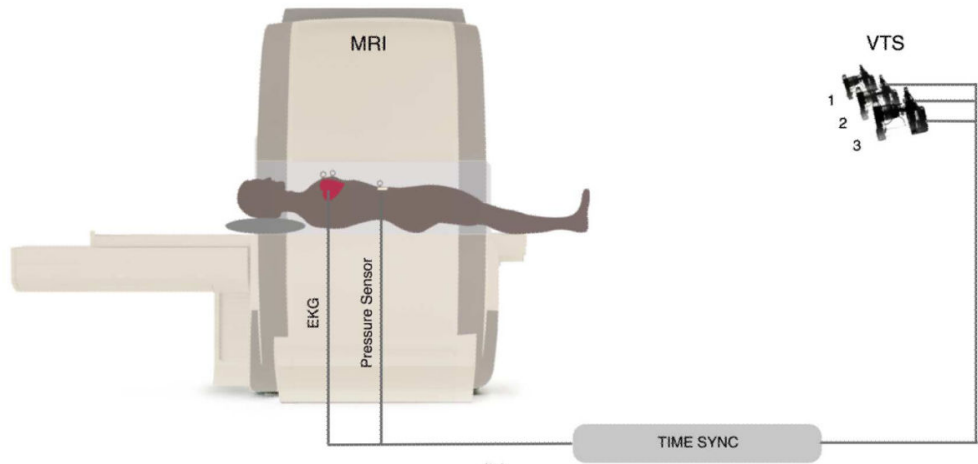

(b)

Fig. 1.

(a) Layout of 7 external marker positions on the chest and abdomen of subjects. (b) Illustration of a subject undergoing imaging positioned in supine and feet-first orientation in the MR scanner with 3 cameras of the VTS employed for tracking external marker motion. The MR scanner and the VTS are temporally synchronized by having a signal from the MRI trigger the start of motion tracking. ECG and respiratory information from the pressure sensor is acquired simultaneously during MRI acquisition. 


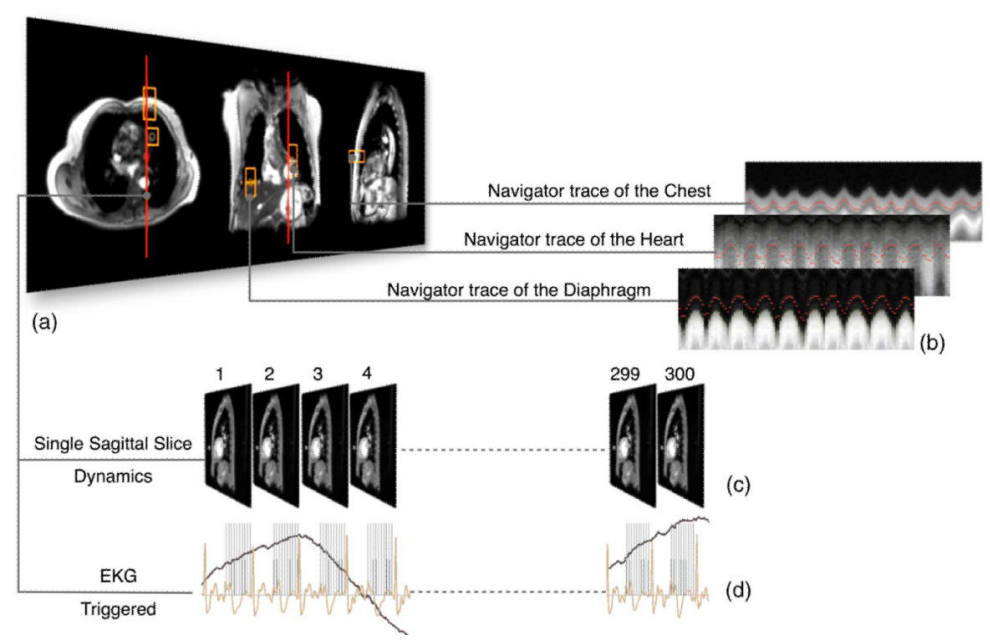

Fig. 2.

(a) Illustrated is the positioning of navigator beams (shown as boxes) on the dome of the right hemi-diaphragm, the superior wall of the left ventricle, and the chest wall. The vertical lines in the transaxial and the coronal slices indicate the position of sagittal slices acquired along with the navigator signals. (b) Shown is example output from the 3 navigator beams as plots of the 1D gray-scale information perpendicular to each body interface versus time. These portray respiratory motion of the chest wall, heart, and diaphragm. (c) Illustrated are the 300 dynamic sagittal slices acquired when the heart was at mid-diastole. (d) Shown at the bottom along with portions of the EKG signal is the signal from the pressure sensor about the abdomen of the volunteers. 


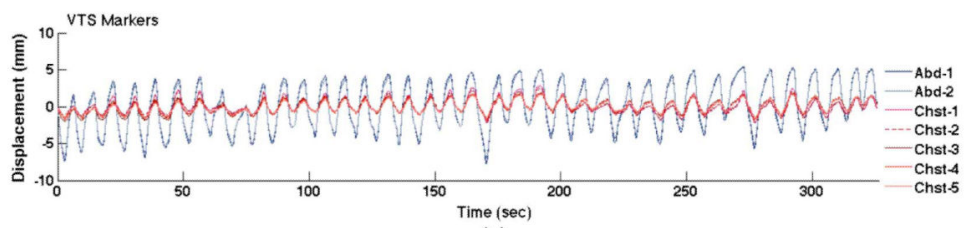

(a)
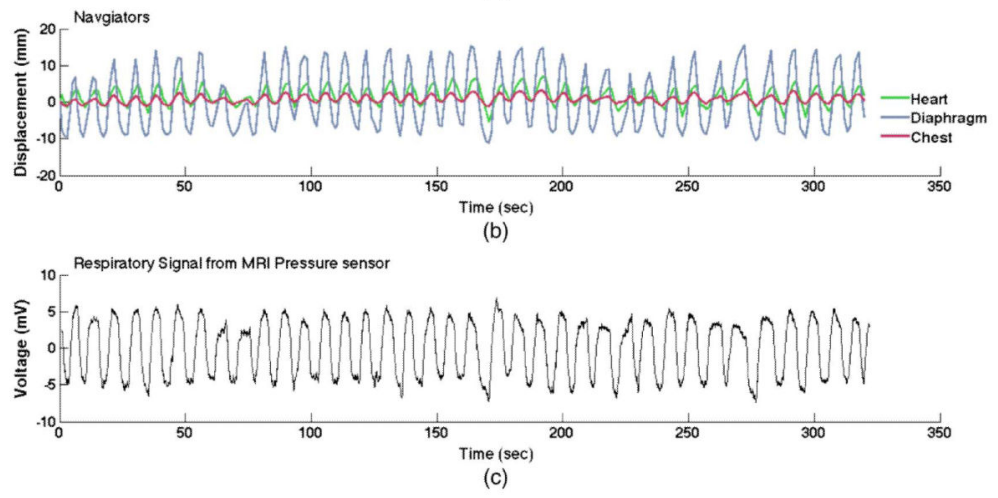

Fig. 3.

(a) Shown is the plot of the displacements of the external chest and abdomen markers as a function of time for about 5 minutes as measured by the VTS for volunteer 3. (b) Plotted are the displacements of the internal heart, diaphragm and chest wall respiratory motions obtained by the respective navigator as a function of time. (c) Plot of the respiratory pressure sensor data during MR acquisition. The plots demonstrate the well defined spatial and temporal correlation between the internal and the external motion for this subject. 


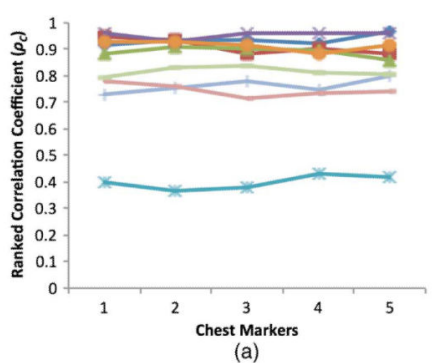

(a)

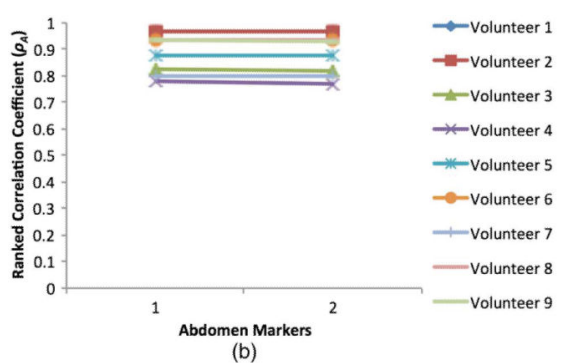

Fig. 4.

Shown are the Spearman ranked correlation coefficients between the MRI Navigator heart motion and the VTS (a) chest and (b) abdomen markers versus marker number for 5 volunteers. 

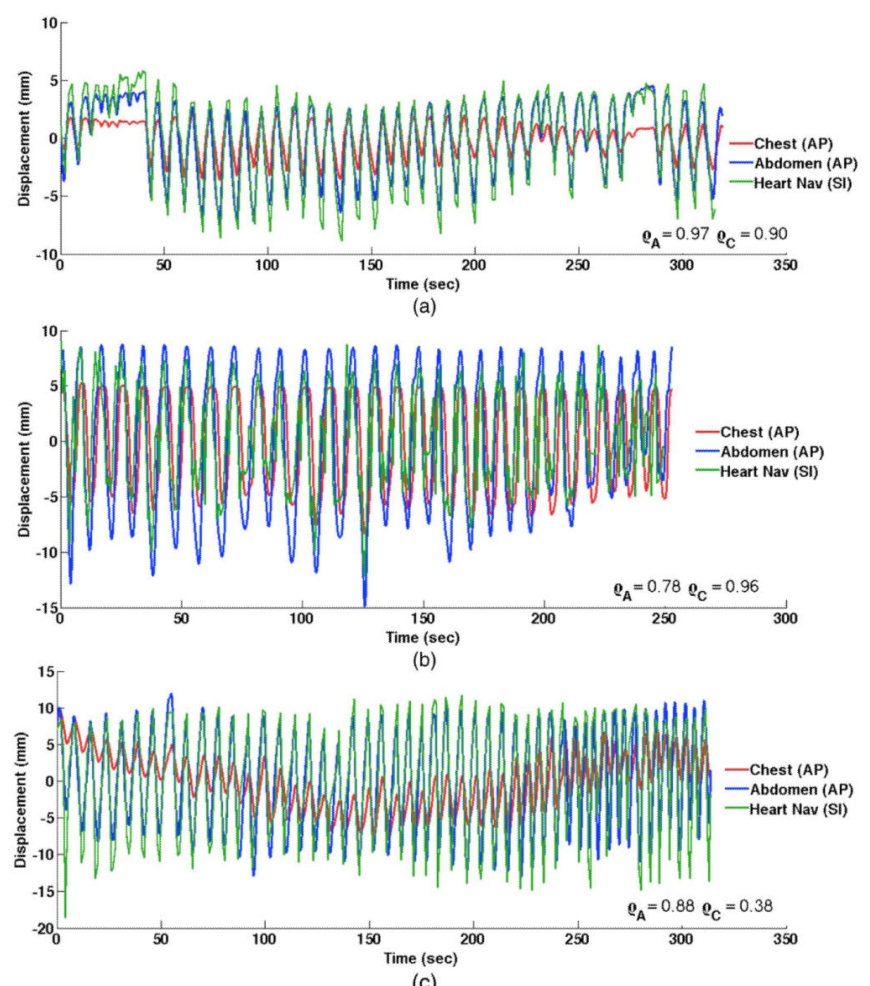

(c)

Fig. 5.

The time series plots for Volunteers 2 (a), 4 (b) and 5 (c) show the VTS determined AP displacements of selected external chest and abdomen markers as a function of time during MRI acquisition. Also shown is the internal SI respiratory motion of the heart superior LV wall as determined by the Navigator. The Spearman ranked correlation coefficients between the MRI Navigator heart motion and the VTS chest $(\rho C)$ and abdomen $(\rho A)$ markers are also shown. 


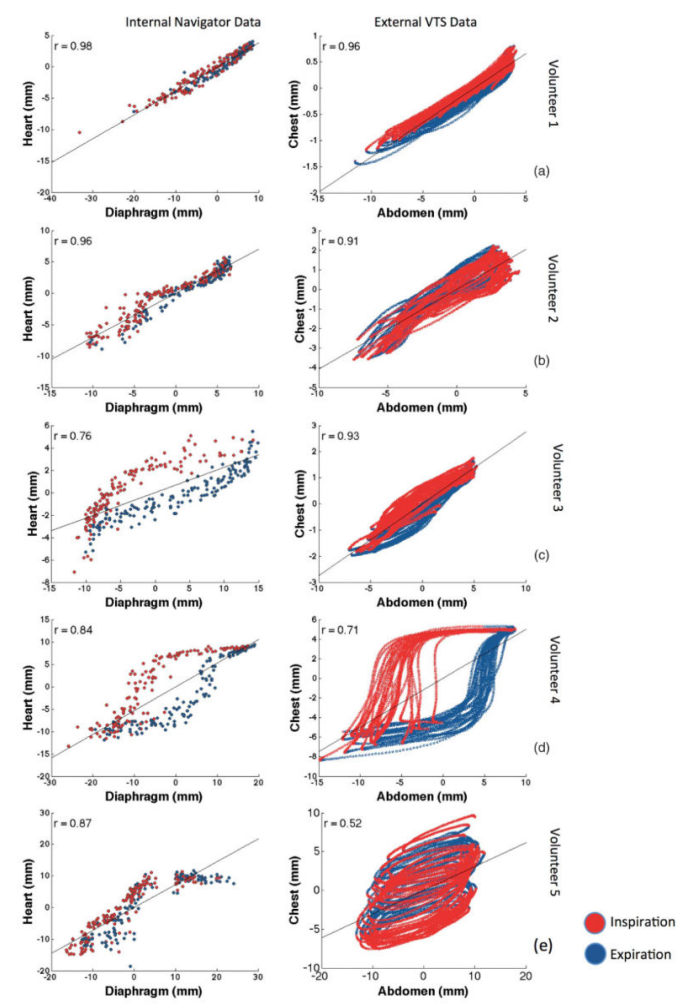

Fig. 6.

Shown in (a) through (e) are 2D scatter plots of pair-wise comparisons of the navigator data for the heart and the diaphragm, and the VTS data for the external chest and abdomen markers for five volunteers. The straight lines are linear fits to the data with Pearson's correlation coefficient $r$ in upper left corner of each plot. The respiratory signals acquired from the navigator and VTS are separated into inspiration and expiration for better visualization of the inspiratory and expiratory trajectories of the heart and external markers. Note scales vary for each plot and that the higher sampling rate of the external markers is evident in the increased density of the points plotted. 

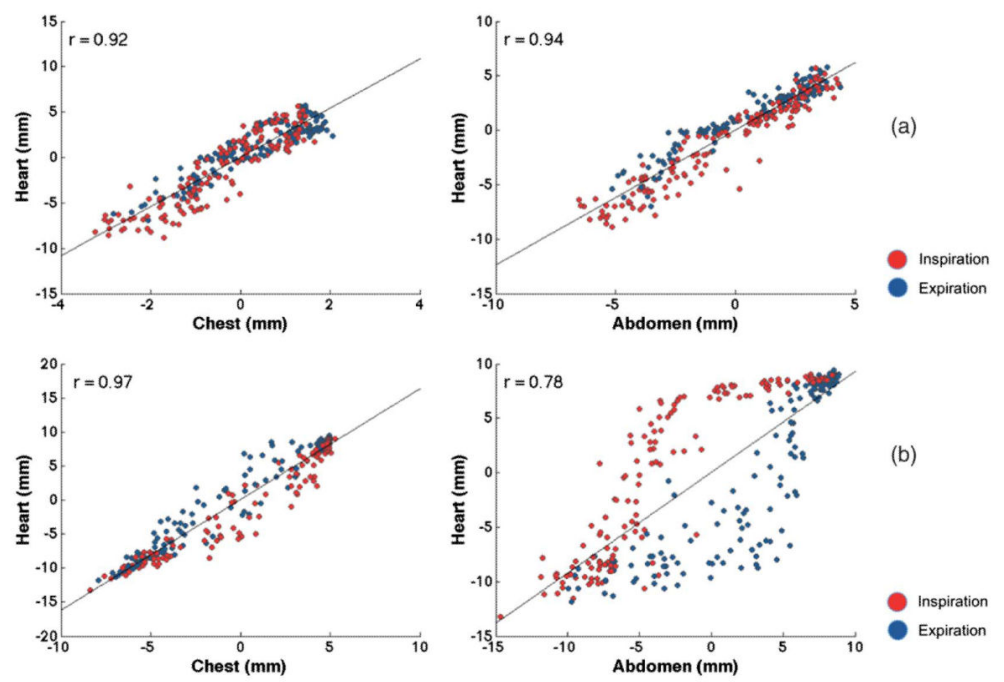

Fig. 7.

2D scatter plots of pair-wise comparisons between the navigator data of the heart and the down-sampled VTS data of the external chest and abdomen markers for two cases: (a) linear pattern (Volunteer 2), and (b) hysteretic pattern (Volunteer 4). The plots illustrate the correlation between the external markers (chest and abdomen) and the internal respiratory motion of the heart. 

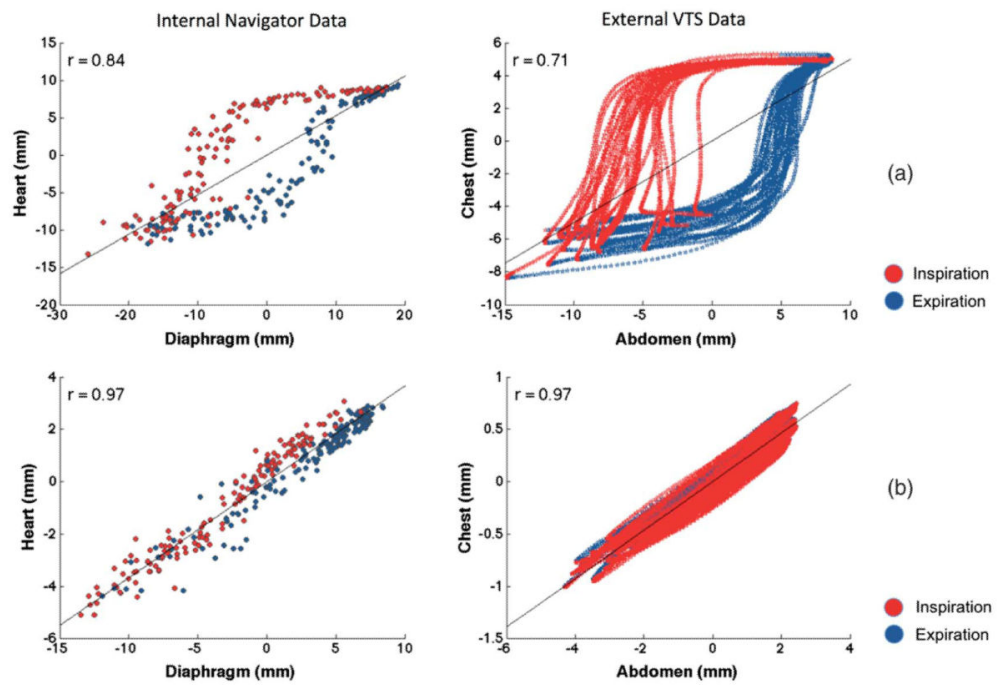

Fig. 8.

2D scatter plots of pair-wise comparisons of the navigator data for the heart and the diaphragm, and the VTS data for the external chest and abdomen markers for one volunteer 4 are shown for (a) deep breathing and (b) shallow breathing acquired on different days. Note how these plots clearly show the hysteretic (a) and linear (b) behavior internally and externally. 